\title{
The Failure of Business Forecasting and Analytical Methods
}

Jarrett JE *

University of Rhode Island, USA

*Corresponding author: Jarret JE, Professor, University of Rhode Island, Ballentine Hall Kingston, RI 02881 USA, Tel: + 4018744169, E-mail:

jeffreyejarrett@gmail.com

Rec date: October 26, 2014, Acc date: October 27, 2014, Pub date: November 15, 2014

Copyright:@ 2014 Jarret JE. This is an open-access article distributed under the terms of the Creative Commons Attribution License, which permits unrestricted use, distribution, and reproduction in any medium, provided the original author and source are credited.

\section{Editorial}

Corporate planning by senior management contains the practice of business and economic forecasting and the analysis of data.

Further it is part practiced heavily by business firms today and agencies of government that act in similar capacities for the general social good of a society or economy. The reasons for this failure are wide and numerous but some essential characteristics are common.

First, forecasting is often given over to middle managers who may be very effective in handling quick changes to detailed plans instantly but are not familiar with the varying methods of forecasting available today in computer software. Skills such as the ability to produce cosmetic and informative spreadsheets are not of use in predicting the economic events that create success/failure in business activities. Success or failure is not always measured in dollars of profit nor in minimization of costs or the ability to supply parts to a finishing process.

Second, standard in today's programs of study at the even the most prestigious graduate programs in business administration no longer have complete courses in forecasting methods and other quantitative tools. The lack of these courses in the curriculum is often the result of many graduate schools promoting MBA, MSA programs in Accounting and/or Finance with no knowledge of decision support systems. These decision support systems include knowledge of forecasting and integrated software to produce forecasts that are based on historical data and knowledge which combines with observed conditional probabilities can produce an optimal result with also valuing the risks associated of making decision without complete knowledge of the future. The consequences of failure or the more likely sub-optimal decisions are not evaluated and sub-standard results occur.

Third, graduate programs are often centered on producing graduate students who may pass a particular certificate examination but have very limited knowledge of how this knowledge fits into the global plan of an organization to produce optimum results. Graduate programs need more emphasis in the mathematics underlying the theory taught in graduate classes on managerial economics, operations research and planning and time series methods for forecasting. Business forecasting is a complex and technical service which requires much more than the ability to produce a "pro-forma" statement or a spreadsheet which answers questions concerning "what-if" a parameter of the decision model changes due to a change in the price of a supply item. Although, this is an important in determining inventory and supply policy, a quantitative inventory and control system may already have accounted for the risks associated with sudden changes in the supply environment.

To give an example of how planning may go wrong, we wish to consider a project that went wrong due to lack of education in business forecasting and data analysis and a comprehensive appreciation of the concepts of prediction and its role in planning. Consider an example from the analysis of data collected through diagnostic tests in a laboratory designed to prevent the spread of infections and diseases contracted by civilians working in a high risk area. Modern bio surveillance involves the monitoring of large amounts and wide ranges of data from samples of diagnostic and prediagnostic data. This purpose is to give health care professionals to recognize, detect, investigate and respond to the outbreaks of disease and epidemics. A central tool in this monitoring in classical disease surveillance migrated to bio surveillance is the use of multivariate quality control methods. Applied multivariate statistical control methods with the application of multivariate quality control (MQC) to syndromic surveillance permit an analyst to focus on directionally sensitive procedures in bio surveillance. Researchers produced one sided multivariate exponentially weighted moving-average (MEWMA) control charts for the analysis of health data. Others used a solution to solve a statistically constrained economic model of a multivariate exponentially moving-average control chart in which external intangible costs were considered. More analysts produced an MQC Bayesian model for bio surveillance and others introduced in practice directionally sensitive MQC charts to bio surveillance methods. They examined four such techniques and came to conclusions based on simulated data, but suggested further research in the application of these methods. For other examples of statistical decision models in monitoring bio surveillance see those who applied adaptive regression to syndromic surveillance data which leads to the discussion of time series models for syndromic surveillance.

I find it difficult to find these problems discussed in MBA and similar programs in the management of the health sciences. We have turned graduate education over to those who are only interested in short-term, quick profit, decision making such as the implementation of Mergers and Acquisition and the like. Although W. Edwards Deming (my professor) warned top executives to focus on their customers and produce products and services that produce high quality output whether it be an end-product or service, we still educate graduates without the knowledge of forecasting, decision-modeling using modern forecasting, data analytical and time series methods. 